\title{
The Application of Molecular Markers to Accelerate the Recovery of Cytoplasmic and Nuclear Male Sterility in South African Onion (Allium cepa L.) Hybrid Parental Lines
}

\author{
Inge Gazendam ${ }^{1}$, Maria M. Greyling ${ }^{1} \&$ Sunette M. Laurie ${ }^{1}$ \\ ${ }^{1}$ Agricultural Research Council-Vegetable and Ornamental Plants (ARC-VOP), Pretoria, South Africa \\ Correspondence: Inge Gazendam, Agricultural Research Council-Vegetable and Ornamental Plants (ARC-VOP), \\ Private Bag X293, Pretoria, 0001 South Africa. Tel: 27-12-808-8000. E-mail: igazendam@arc.agric.za
}

Received: December 3, 2018

Accepted: March 5, $2018 \quad$ Online Published: June 15, 2018

doi:10.5539/jas.v10n7p95

URL: https://doi.org/10.5539/jas.v10n7p95

\begin{abstract}
Male sterility is important to prevent self-pollination and loss of the onion hybrid genotype. Classic methods require 4-8 years of progeny testing before the cytoplasm type can be determined. An accurate and time-saving method was needed. Various types of markers were tested for application to South African onion parental lines of hybrid cultivars, and which could determine male sterility and maintainer genotypes accurately and easily with large numbers of samples. Five cytoplasmic (5'cob, orfA501, orf725, IGS and cob-type 2) and four nuclear markers (jnurf13, isotig34671_610, isotig30856_1351 and isotig29186_1830) were sourced. Genomic DNA was isolated from onion seedlings and young leaves growing from bulbs in the Agricultural Research Council (ARC) research field. PCR marker amplification products were separated by agarose or denaturing polyacrylamide gel electrophoresis (PAGE) gels. Real-time polymerase chain reaction (PCR) was performed with custom TaqMan ${ }^{\circledR}$ SNP genotyping assays containing primer/probe pairs designed to detect single nucleotide polymorphisms (SNPs) linked to the nuclear Ms locus. OrfA501 proved useful as a presence/absence marker for cytoplasmic male sterility, while TaqMan ${ }^{\circledR}$ SNP genotyping assays were superior to the jnurf13 nuclear marker in terms of rapid throughput. PCR molecular markers and custom TaqMan ${ }^{\circledR}$ SNP genotyping assays were efficient in screening the onion lines rapidly and accurately for their cytoplasmic and nuclear male sterility genotype. These methods reduced the time to identify the correct genotype of male sterile and maintainer lines, gave accurate genotypic information and proved to be useful on a larger scale. These molecular marker methods will facilitate the production of the correct seed for commercialization of onion lines worldwide.
\end{abstract}

Keywords: cytoplasmic male sterility, onion, real-time PCR, Taqman ${ }^{\circledR}$ SNP genotyping assay

\section{Introduction}

Cytoplasmic male sterility (CMS) is widely used in plant breeding schemes to produce $\mathrm{F}_{1}$ hybrid cultivars. It has been discovered in 271 plant species to date, and is the result of male-sterility-inducing factors present mostly in the mitochondrial genome causing the inability to produce viable pollen grains (Gai \& Meng, 2010). Cytoplasmic male sterility is therefore inherited maternally.

Onion (Allium cepa L.) has been used as food for centuries and is an important vegetable crop grown worldwide. It is used for flavouring food, both at the mature and immature stages, and used in salads and are pickled (Brewster, 2008). The male sterility system used in onion hybrid development is controlled by a dual system of CMS and nuclear genes (Havey, 2000). Two CMS systems have been discovered in onion, CMS-S and CMS-T, but only the CMS-S type is widely used in breeding schemes, since it has only a single nuclear locus that can restore male sterility caused by the CMS-S cytoplasm (restorer-of-fertility locus MS), and therefore has a simple inheritance pattern. The CMS-S system is also stable over diverse environmental conditions (Havey, 2000). The CMS-T system is influenced by three independently segregating restorer loci, with dominant alleles restoring fertility in specific combinations (Havey, 2000). This makes selection and breeding for onion male sterility using the CMS-T system more complicated, therefore it is not as widely used compared to CMS-S.

In South Africa, the onion breeding program commenced during the 1950's at the Department of Agriculture's research component based at Roodeplaat (currently named the Agricultural Research Council (ARC)). Male sterility was introgressed into the local material for the first time during the late 1980's. The main aim of 
breeding was the development of short day onion lines and test $F_{1}$ hybrids with long storability, multiple bulb scales and high bulb firmness, with adaptation to local conditions. To achieve this aim, short day lines were crossed with intermediate day lines, which inherently had the mentioned bulb quality traits. This was followed by a number of years of selection against bolting and splitting. The ARC male sterile A-lines (msms nuclear gene; cytoplasmic factor $S$ ) and $\mathrm{F}_{1}$ test hybrids were made available to the commercial seed companies on contract for possible commercialization of $\mathrm{F}_{1}$ hybrids.

The seed companies only received seed of the male sterile A-line and C-line (cultivar that acts as a pollen parent) for hybrid seed production, while the ARC retains the maintainer B-line ( $m s m s$ nuclear gene; cytoplasmic factor $N$ ), giving the ARC the ability to control the usage of the materials. While maintaining or producing seed of the male sterile parent, there can be complications, for example, when the nuclear $M s$ allele finds its way into the maintainer B-line. $M s$ is dominant and causes fertility restoration. The production of pure $\mathrm{F}_{1}$ hybrid seed is then compromised. This will lead either to complaints or claims by the farmers using the seed. The problem can be counteracted to a certain extent by rogueing fertile A-line plants at any early stage. However, this does cause a loss of seed production. Likewise, mixing of bulbs can result in $N$-cytoplasm in the male sterile A-line. $S$-cytoplasm in the B-line can generally be removed by rogueing, as the bulbs are male-sterile, unless masked by the nuclear fertile genotype $M s$.

Recovery of the correct genotype in the A and B lines is thus critical. However, it requires 4-8 years of progeny testing before the cytoplasm type can be determined (Brewster, 2008). This is an expensive, time consuming and a very exacting process. Melgar and Havey (2010) further noted less than $100 \%$ male fertility restoration when test crossing the dominant $M s$ with male sterile plants, thus it may even require that scoring should be done over a number of years to more confidently identify the correct genotypes. Failures lead to inbreeding and eventual loss of the maintainer B-lines. Without the maintainer line the male sterile A-line cannot be produced, and as a result the commercial $\mathrm{F}_{1}$ hybrid cultivar is lost.

Accurate and time-saving methods were sought by various researchers to shorten the time period needed to determine the male sterility genotype of different onion lines. Various PCR and RFLP molecular markers have been documented in the literature for cytoplasmic male sterility. These rely on the variation of relative copy numbers of male-sterility inducing mitochondria (Sato, 1998; Engelke, Terefe, \& Tatlioglu, 2003; Kim et al., 2009; Kim \& Yoon, 2010), or polymorphic chloroplast sequences (Havey, 1995; Kim, 2014). A PCR marker linked to the nuclear Ms locus, jnurf13, was documented by Kim (2014). SNP markers near the nuclear Ms locus were furthermore genotyped using the Roche KASPar assay (Duangjit, Bohanec, Chan, Town, \& Havey, 2013; Havey, 2013). The top three SNP markers (isotig34671_610, isotig30856_1351 and isotig29186_1830) showed less than $30 \%$ recombination $(11,13$, and $15.7 \%$, respectively) between the recessive $m s$ allele and each SNP among random plants collected from three open-pollinated populations of onion. Most authors screened onion lines with only one or a few molecular markers.

The present study is aimed at testing the various available molecular markers to accelerate recovery of male sterile-maintainer parental lines used in the ARC hybrid seed production. The rationale was to compare these markers and see if they correspond to each other when applied to the ARC population of onion parental lines, and to determine which is most effective to easily and confidently determine male sterile genotypes for a large sample size. TaqMan ${ }^{\circledR}$ SNP genotyping assays, designed to detect SNPs linked to the nuclear Ms locus, were also employed to improve the speed of throughput compared to the jnurfl 3 nuclear marker.

\section{Material Studied}

Preliminary testing of markers was conducted on seedlings, while the F1 parental lines, mentioned below, were in the bulb storage phase (Feb-May 2015). Seeds of putative reference lines (Lines 1 and 2 = male sterile lines; Lines 3 and $4=$ maintainer lines) were sown in a seedling tray filled with Hygromix (Hygrotech Pty Ltd South Africa) in a greenhouse. The first mature leaf ( $\pm 80 \mathrm{~mm}$ in length) of 10 replicates of each line were sampled by cutting them individually into small pieces into a microfuge tube. The samples were kept on ice until genomic DNA was extracted.

After obtaining the results from above, five sets of male sterile-maintainer combinations produced by the ARC were tested, namely, R601A (seed lot S11-11) and R601B (S11-14), R801A (S12-005) and R801B (S12-006), R1001A (S09-003) and R1001B (S09-004), R1201A (S09-001) and R1201B (S09-002), and R1401A (S9-09) and R1401B (S9-10). Seeds were sown in February 2014 for bulb harvesting in October 2014 at Roodeplaat, Pretoria $\left(25.60^{\circ} \mathrm{S}, 28.34^{\circ} \mathrm{E}\right)$. The bulbs were stored until May 2015 , selected for bulb quality according to the specific line and planted in the field for seed production. Unfortunately, no bulbs of R1401B survived during the storage period. At the start of flowering, leaf samples were collected from 20 randomly selected individuals of 
each of the nine lines. The apical section $( \pm 50 \mathrm{~mm}$ in length) of the youngest leaf of the plant was collected in each case and handled as above.

For larger scale genotyping with the molecular markers, bulbs of four ARC onion lines (R101B, seed lot S03-2; R301A, seed lot S07-3; and R301B, seed lots S07-4 and S09-4) were produced during 2015 for seed production in 2016. The bulbs were stored until May 2016, selected for bulb quality according to the specific line and planted in the field for seed production. Leaf samples were collected as above in July 2016 from all 745 individually labelled onion plants for genomic DNA isolations.

\section{Methods}

\subsection{Genomic DNA Isolation}

Genomic DNA was extracted following a modified CTAB protocol. The method from Murray and Thompson (1980) was modified for plants rich in polysaccharides and polyphenols (Moyo, Amoo, Bairu, Finnie, \& Van Staden, 2008; Sharma \& Purohit, 2012). The CTAB concentration of the isolation buffer was increased to $2.5 \%$, $\mathrm{NaCl}$ from $1.4 \mathrm{M}$ increased to $2 \mathrm{M}$, and 3\%(w/v) PVPP added to the plant material before grinding with carborundum. The mixture, after incubation at $60{ }^{\circ} \mathrm{C}$ for $30 \mathrm{~min}$, was extracted with an equal volume of chloroform: isoamyl alcohol $(24: 1) .1 / 10^{\text {th }}$ volume $10 \% \mathrm{CTAB} / 0.7 \mathrm{M} \mathrm{NaCl}$ was added to the recovered supernatant, mixed gently and extracted again. Genomic DNA was precipitated with 1 volume ethanol at room temperature and collected by centrifugation at $500 \times \mathrm{g}$ for $5 \mathrm{~min}$. The pellet was dissolved in high salt TE (TE with $1 \mathrm{M} \mathrm{NaCl})$ and treated with RNaseA $(2.5 \mu \mathrm{lof} 10 \mathrm{mg} / \mathrm{ml})$ at $37{ }^{\circ} \mathrm{C}$ for $3 \mathrm{~h}$ and re-precipitated with 0.6 volumes isopropanol at room temperature. The pellet was washed with $70 \%$ and $100 \%$ ethanol, respectively, air dried and resuspended in TE buffer at room temperature overnight. DNA was quantified on a Nanodrop ND-1000 spectrophotometer (Thermo Scientific) and stored at $-20^{\circ} \mathrm{C}$. Dilutions of $10 \mathrm{ng} / \mu \mathrm{l}$ were prepared in $\mathrm{sdH}_{2} \mathrm{O}$ before using them as templates in PCR.

\subsection{PCR Molecular Markers for Determination of Cytoplasmic and Nuclear Male Sterile Genotypes}

Five cytoplasmic (5'cob, Sato, 1998; orfA501, Engelke et al., 2003; orf725, Kim et al., 2009; IGS, Kim, 2014; cob-type 2, Kim \& Yoon, 2010) and four nuclear markers (jnurf13, Kim, 2014; isotig34671_610, isotig30856_1351 and isotig29186_1830, Duangjit et al., 2013; Havey, 2013) selected from the literature were tested. The gene region, organelle genome origin, literature reference, PCR primer sequences, primer annealing temperature $\left(\mathrm{T}_{\mathrm{m}}\right)$ and expected amplification product expected for each genotype (cytoplasmic $N, T$ and $S$ or nuclear $M s$ or $m s$ ) are indicated in Appendix A.

PCR was carried out on $30 \mathrm{ng}$ of genomic DNA in $200 \mu \mathrm{l}$ PCR tubes using $0.5 \mathrm{U}$ of TaKaRa Taq (TAKARA BIO INC., Otsu, Shiga, Japan), $1 \times$ buffer, $3.5 \mathrm{mM} \mathrm{MgCl} 2,200 \mu \mathrm{M}$ of each dNTP, $0.24 \mu \mathrm{M}$ of each primer in a total volume of $12.5 \mu \mathrm{l}$. The cycling conditions included denaturation at $94{ }^{\circ} \mathrm{C}$ for $2 \mathrm{~min}$, cycling 30 times at $94{ }^{\circ} \mathrm{C}$ for $30 \mathrm{~s}$, primer annealing at the temperature as indicated in Appendix A for $30 \mathrm{~s}$ and extension at $72{ }^{\circ} \mathrm{C}$ for $45 \mathrm{~s}$. All products were fully extended by a final elongation step of $7 \mathrm{~min}$ at $72{ }^{\circ} \mathrm{C}$. Large scale PCR screening was performed in 96-well PCR plates and included $10 \mathrm{ng}$ gDNA as template. The reaction volume was decreased to $10 \mu \mathrm{l}$, and contained $0.4 \mathrm{U}$ of Taq per reaction.

PCR amplification products of all markers except jnurf 13 were separated by $1.5 \%$ agarose $/ 0.5 \times$ TAE gel (containing $0.1 \mu \mathrm{g} / \mathrm{ml}$ Ethidium Bromide) electrophoresis at $120 \mathrm{~V}$ for $70-90 \mathrm{~min}$ and visualised on UV light and photographed. The molecular weight marker (MWM) VI (Roche Diagnostics) at $400 \mathrm{ng}$ per lane was utilised to size the fragments. Large scale PCR products of orfA501 were analysed on an Electro-Fast ${ }^{\circledR}$ (ABgene) stretch agarose gel $(1.5 \% \mathrm{w} / \mathrm{v})$ at $100 \mathrm{~V}$ for $30 \mathrm{~min}$. ReddyRun ${ }^{\mathrm{TM}}$ loading dye (ABgene) was added to each PCR reaction to $1 \times$ concentration, and $5 \mu \mathrm{l}$ of the mix loaded onto the gel. Electro-Fast ${ }^{\circ}$ marker $(3 \mu 1,25 \mathrm{ng} / \mu \mathrm{l})$ was loaded in the first well of every second row. Results were scored as cytoplasmic $S$ if a band was present. PCR amplification products of jnurf13 were separated by denaturing $6 \% \mathrm{PAGE} / 8 \mathrm{M}$ Urea/1× TBE gel electrophoresis at $70 \mathrm{~mA}(1.1-1.5 \mathrm{kV})$ for 2.5 hours. Fragments were visualised by silver nitrate staining, the gel dried and photographed. Fragments were sized according to 10bp and MWM V (Roche Diagnostics) DNA ladders.

\subsection{TaqMan ${ }^{\circledR}$ SNP Genotyping Assay for the Detection of SNP Linked to the Nuclear Male Sterility Factor}

Allelic discrimination of three SNPs in linkage disequilibrium with the nuclear restorer of male sterility gene (Ms locus) (Duangjit et al., 2013; Havey, 2013) was assessed using custom TaqMan ${ }^{\circledR}$ SNP genotyping assays (Applied Biosystems), following the same method as was applied by Cho et al. (2006) for the chloroplast $p s b A$ gene. The SNP sequences, primer and probe binding sites are presented in Appendix B.

Real-time PCR was performed on the Rotorgene-3000 (Corbett Life sciences, Qiagen) using a 2-step protocol. Reaction components included $50 \mathrm{ng}$ onion gDNA, 1X TaqMan ${ }^{\circledR}$ genotyping master mix and $1 \mathrm{X}$ SNP 
genotyping assay mix, as per manufacturer's instructions (Applied Biosystems), with a total reaction volume of $15 \mu \mathrm{l}$ in a $100 \mu \mathrm{l}$ Rotorgene tube. The enzyme was activated at $95{ }^{\circ} \mathrm{C}$ for 10 minutes, followed by 46 cycles of $92{ }^{\circ} \mathrm{C}$ for $15 \mathrm{~s}$ and $60{ }^{\circ} \mathrm{C}$ for $60 \mathrm{~s}$. Released dye were detected after each cycle by acquiring 6-FAM fluorescence (for detecting $m s$ ) at 470/510 nm and JOE fluorescence (corresponding to the VIC reporter dye) (for detecting $M s)$ at 530/555nm excitation/emission wavelength, respectively (Appendix B). Allelic discrimination and scatter plot analysis was performed in the Rotorgene software to assign genotypes to the samples, and this was compared to the genotypes obtained with the nuclear PCR marker jnurf13.

\subsection{Phenotyping of the Fertility of Onion Lines}

The fertility phenotype of each of the sampled plants was scored as the presence or absence of pollen of mature onion flowers by visual inspection in the field. The observed phenotype was compared to the expected fertility, which was predicted as a combination of the cytoplasmic and nuclear male sterility genotypes, as determined with PCR and TaqMan ${ }^{\circledR}$ molecular marker screening.

\section{Results}

\subsection{Preliminary Testing of PCR Molecular Markers for Determination of Male Sterile Genotypes}

Five cytoplasmic PCR molecular markers (5'cob, Sato, 1998; orfA501, Engelke et al., 2003; orf725, Kim et al., 2009; IGS, Kim, 2014; cob-type 2, Kim \& Yoon, 2010) were selected from the literature to facilitate accurate and rapid genotyping of onion cytoplasmic male sterility genotypes. Similarly, four nuclear molecular markers, one being a PCR marker (jnurf13, Kim, 2014) and three based on SNPs linked to the restorer-of-fertility locus Ms (isotig34671_610, isotig30856_1351 and isotig29186_1830, Duangjit et al., 2013; Havey, 2013) were selected from the literature to facilitate accurate and rapid genotyping of onion nuclear male sterility genotypes. The gene region, organelle genome origin, literature reference, PCR primer sequences, primer annealing temperature $\left(\mathrm{T}_{\mathrm{m}}\right)$ and expected amplification product expected for each genotype (cytoplasmic $N, T$ and $S$ or nuclear $M s$ or $m s$ ) are indicated in Appendix A.

PCR molecular markers were first tested on eight seedlings each of the reference lines expected to be male sterile (Lines 1 and 2; seed lots S07-3 and S09-003, of onion lines R301A and R1001A, respectively) and maintainer lines (Lines 3 and 4; seed lots S07-4 and S09-4, respectively, of onion line R301B). The appearance of fragments generated by the different PCR markers, and the genotyping results, are presented in Figure 1. The presumed reference onion lines 1, 3 and 4 did not show the expected genotypes.

The 5'cob marker (Sato, 1998) amplified faint 180bp products in all onion samples (Figure 1; 5'cob), but this fragment was stronger in samples designated as cytoplasmic $N$ (Figure 1, line 1: samples 5 and 7, lines 3 and 4: all samples except 6 and 7, respectively). The 414bp fragment was observed in all samples designated as $S$ and absent in all samples designated as $N$.

The orfA501 marker (Engelke et al., 2003) successfully amplified a 473bp chimerical orfA501 fragment associated with $S$ or $T$ cytoplasm (another system of male sterility not commonly used in breeding) in all onion samples designated as $S$ (Figure 1; orfA501; line 1: all samples except 5 and 7; line 2: all samples; lines 3 and 4: only samples 6 and 7, respectively). Sample 8 of line 1 did not initially show a fragment with marker orfA501 (Figure 1, cytoplasmic genotype $\mathrm{S}^{*}$ ), and was attributed to omitted gDNA template in the PCR. This sample was re-run with marker orfA501 in the next analysis and an $S$ genotype was detected.

Specific primers for normal coxl (cox1-F and cox1-R) yields an 833bp fragment for $N$-cytoplasm, but primers for $\operatorname{orf725}$, expected in $S$ and $T$-cytoplasm, yields a 628bp fragment (Kim et al., 2009). $N$-fragments were observed in all onion samples (Figure 1; orf725), even faintly for $S$-samples of lines 1 and 2 (Figure 1; orf725) and the single $S$ cytoplasm samples of lines 3 and 4 (Figure 1; orf725, line 3 sample 6 and line 4 sample 7 , respectively), but samples designated as $N$ only produced the $833 \mathrm{bp}$ fragment, as expected (Figure 1, line 1: sample 5 and 7, lines 3 and 4: all samples except line 3 sample 6 and line 4 sample 7, respectively).

The smaller $S$-cytoplasm IGS fragment (299bp; Havey, 1995; Kim, 2014) was produced from all onion samples designated as $S$ (Figure 1; IGS; line 1: all samples except 5 and 7; line 2: all samples; lines 3 and 4: only samples 6 and 7, respectively). The larger IGS fragment (388bp) was produced from all the other onion samples designated as $N$.

The cob-type 2 marker did not produce cytoplasmic $S$-specific fragments of the expected 852bp size (Kim \& Yoon, 2010) from any of the lines tested (results not shown). Faint non-specific amplification products of $\sim 1000 \mathrm{bp}$, and strong amplification products of $\sim 2100 \mathrm{bp}$ were observed for some lines. Specificity was enhanced by increasing the $\mathrm{Tm}$ from $55^{\circ} \mathrm{C}$ to $58^{\circ} \mathrm{C}$, but it did not make a difference. 
The jnurf13 marker is a codominant marker (Kim, 2014) and could detect the homozygous recessive (msms), heterozygous $(M s m s)$ and homozygous dominant $(M s M s)$ nuclear genotypes of four selected onion lines. Only line 2 was 100\% homozygous recessive (msms) as expected for A and B lines (Figure 1; jnurfl3; line 2), but the rest were segregating for the fertility restoring $M s$ allele.

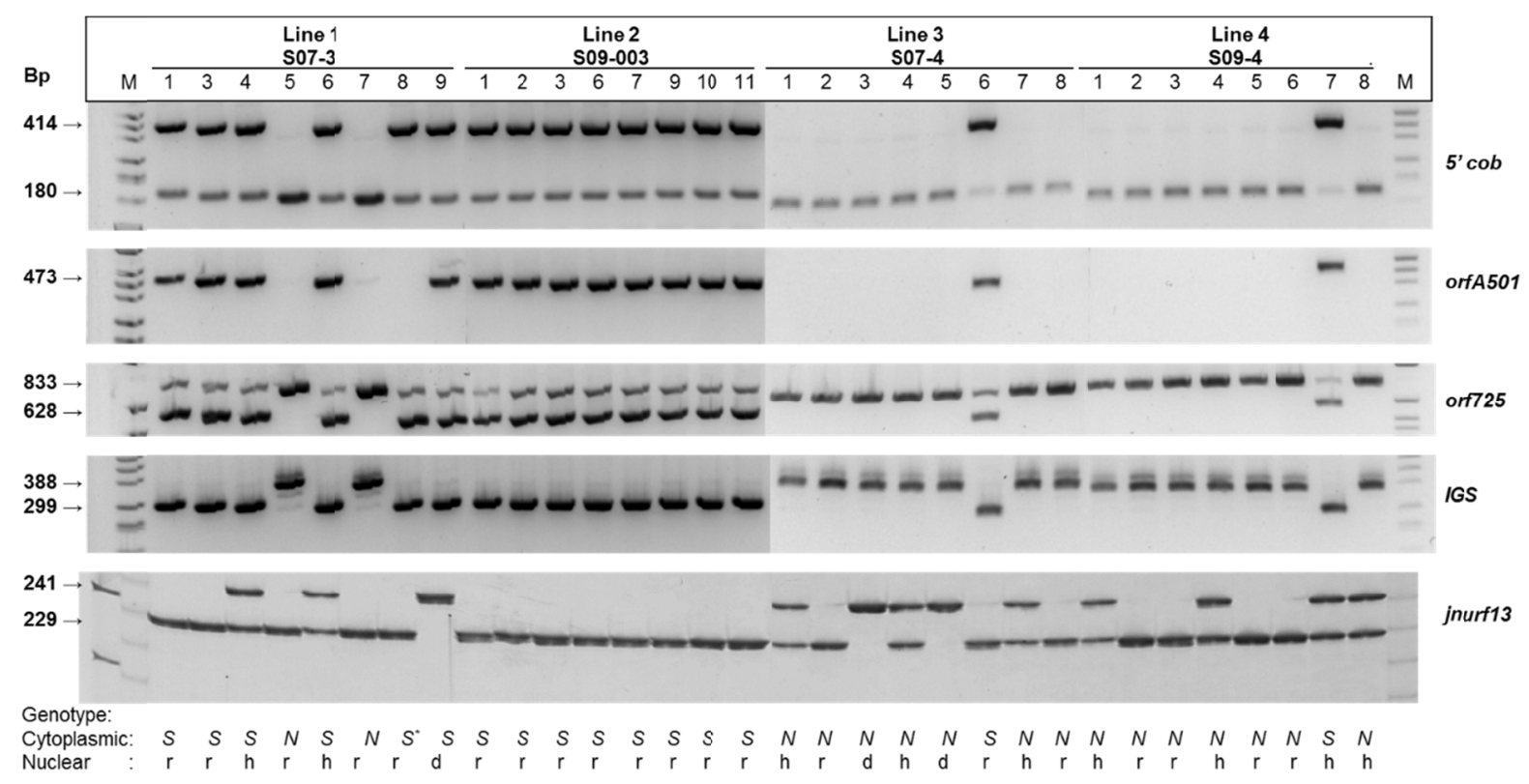

Figure 1. Amplification products generated by five different PCR markers on four ARC reference onion lines

Fragment sizes are indicated in base pairs on the left, and the gene region targeted with the PCR marker on the right. The four lines, each with eight individual plant replicates, are labelled on top. M: MWM VI (Roche) except at jnurf13: 10bp ladder (Roche). The cytoplasmic genotype ( $S=$ sterile, $N=$ normal) and nuclear genotype $(\mathrm{r}=$ homozygous recessive $(m s m s), \mathrm{h}=$ heterozygous $(M s m s), \mathrm{d}=$ homozygous dominant $(M s M s)$ ) of each sample is indicated at the bottom. $S^{*}$ denotes the cytoplasmic genotype of line 1 sample 8 , which did not initially show a fragment with marker orfA501. However, an $S$ genotype was detected when this sample was re-run with marker orfA501 in the next analysis (results not shown). This was attributed to omitted gDNA template in the first PCR reaction.

\subsection{TaqMan ${ }^{\circledR}$ SNP Genotyping Assays for Determination of Male Sterility Genotypes}

The top three SNP markers (isotig34671_610, isotig30856_1351 and isotig29186_1830; Duangit et al., 2013; Havey, 2013) were developed into TaqMan ${ }^{\circledR}$ SNP genotyping assays. They are linked to the Ms locus with 11, 13 and $15.7 \%$ recombination, respectively, between the recessive $m s$ allele and each SNP among random plants collected from three open-pollinated populations of onion. The three TaqMan ${ }^{\circledR}$ SNP genotyping assays (A: isotig34671_610; B: isotig30856_1351 and C: isotig29186_1830; Appendix B) were validated against known nuclear male sterility genotypes of eight S03-2 samples (earlier seed lot from R101B). TaqMan ${ }^{\circledR}$ SNP genotyping assay results (points on a scatter plot, Figure 2, with different colours for each assay, namely A: isotig34671_610 (blue); B: isotig30856_1351 (pink) and C: isotig29186_1830 (green)) assigned the same nuclear genotypes to samples as obtained with the jnurf13 PCR marker. 


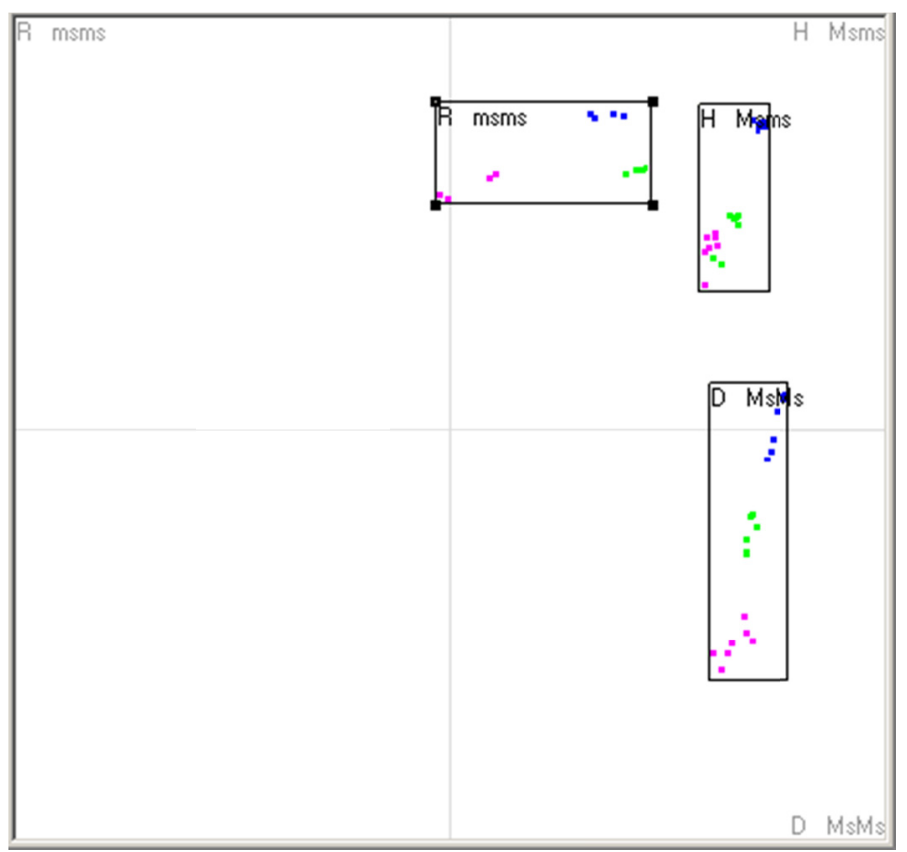

Figure 2. Scatter plot analysis of TaqMan ${ }^{\circledR}$ SNP genotyping assay results for three SNPs in linkage disequilibrium with the $m s$ allele of onion, applied to eight samples of S03-2 genotyped previously with jnurf 13 . Samples are colour coded according to the TaqMan ${ }^{\circledR}$ SNP genotyping assay used: A (isotig34671_610): blue; B (isotig30856_1351): pink; C(isotig29186_1830): green

\subsection{Identification of the Cytoplasmic and Nuclear Genotype of Onion Breeding Pairs}

The PCR marker results and allelic discrimination by TaqMan ${ }^{\circledR}$ SNP genotyping assays with regards to cytoplasmic and nuclear genotype of nine F1 hybrid parental lines grown in 2015 are summarised in Table 1. Only two samples out of 232 (s 74 and s 80 ) were excluded due to low quality genomic DNA, resulting in ambiguous PCR results. The cytoplasmic male sterility genotypes are represented by a combination of the four cytoplasmic PCR marker results. There was only one sample where all four cytoplasmic markers did not give the same genotype. The orfA501 marker genotyped sample 6 of R601B (s 26) as $S$, while the other three markers showed an $N$ genotype (results not shown). Nuclear genotypes, determined with TaqMan ${ }^{\circledR}$ SNP genotyping assays A, B and C and marker jnurf13, were identical for all parental lines tested except one (R601, see discussion). The expected fertility was determined as a combination of the cytoplasmic and nuclear male sterility genotypes, and compared to the observed fertility status as scored by the presence of pollen in mature onion flowers. The observed fertilities of F1 hybrid parental lines R601B, R801A and B, R1201A and R1401A in the field were as expected. 
Table 1. Cytoplasmic and nuclear male sterility genotypes of nine South African onion lines growing from bulbs in the field in 2015. Five PCR molecular markers and three TaqMan ${ }^{\circledR}$ SNP genotyping assays were applied to selected individual plants per line to determine their cytoplasmic and nuclear male sterility genotypes. Results not as expected are indicated in italics

\begin{tabular}{|c|c|c|c|c|c|c|c|c|}
\hline \multirow{2}{*}{$\begin{array}{l}\text { Parental } \\
\text { line }\end{array}$} & \multicolumn{4}{|c|}{$\begin{array}{l}\text { Marker and nr individuals } \\
\text { per nuclear genotype }\end{array}$} & \multirow{2}{*}{$\begin{array}{l}\begin{array}{c}\text { Cytoplasmic } \\
\text { genotype }\end{array} \\
\text { Combination of } \\
4 \text { cytoplasmic } \\
\text { PCR markers }\end{array}$} & \multirow{2}{*}{$\begin{array}{l}\text { Expected } \\
\text { fertility }\end{array}$} & \multirow{2}{*}{$\begin{array}{l}\text { Observed } \\
\text { fertility in field }\end{array}$} & \multirow{2}{*}{ Result } \\
\hline & $\begin{array}{l}\text { TaqMan }{ }^{\circledR} \\
\text { SNP assay A }\end{array}$ & $\begin{array}{l}\text { TaqMan }{ }^{\circledR} \\
\text { SNP assay B }\end{array}$ & $\begin{array}{l}\text { TaqMan }{ }^{\circledR} \\
\text { SNP assay C }\end{array}$ & $\begin{array}{l}\text { PCR marker } \\
\text { jnurf13 }\end{array}$ & & & & \\
\hline R601A & msms: $8 / 8$ & $\begin{array}{l}\text { MsMs: } 6 / 20 \\
\text { Msms: } 10 / 20 \\
\text { msms: } 4 / 20\end{array}$ & msms: $8 / 8$ & msms: $8 / 8$ & $\begin{array}{l}N: 1 / 20 \\
\text { (sample 13) } \\
\text { S: } 19 / 20\end{array}$ & $\begin{array}{l}\text { 1/20 (assay } \\
\text { B genotype } \\
\text { disregarded) }\end{array}$ & $0 / 20$ & $\begin{array}{l}\text { No (1 mixed } \\
\text { sample, 13) }\end{array}$ \\
\hline R601B & msms: 72/72 & $\begin{array}{l}\text { MsMs: } 20 / 64 \\
\text { Msms: } 31 / 64 \\
\text { msms: } 13 / 64\end{array}$ & msms: $8 / 8$ & msms: $40 / 40$ & $\begin{array}{l}\mathrm{N}: 72 / 72 \\
\text { (Sample } 6 \text { is } \\
\text { S with only } \\
\text { orfA501 marker) }\end{array}$ & $\begin{array}{l}20 / 20 \\
\text { (disregard } \\
\text { sample } 6 \\
\text { orfA501 result) }\end{array}$ & $\begin{array}{l}17 / 17 \\
\text { (3 plants didn't } \\
\text { have seed stalks) }\end{array}$ & Correct \\
\hline R801A & msms: $8 / 8$ & msms: $20 / 20$ & msms: 7/7 & msms: $8 / 8$ & S: $20 / 20$ & $0 / 20$ & $0 / 20$ & Correct \\
\hline R801B & msms: $9 / 9$ & msms: $18 / 18$ & msms: $5 / 5$ & msms: $10 / 10$ & $\mathrm{~N}: 18 / 18$ & $18 / 18$ & $18 / 18$ & Correct \\
\hline R1001A & $\begin{array}{l}\text { Msms: } 2 / 8 \\
\text { msms: } 6 / 8\end{array}$ & $\begin{array}{l}\text { Msms: } 7 / 20 \\
\text { msms: } 13 / 20\end{array}$ & $\begin{array}{l}\text { Msms: } 2 / 8 \\
\text { msms: } 6 / 8\end{array}$ & $\begin{array}{l}\text { Msms: } 2 / 8 \\
\text { msms:6/8 }\end{array}$ & S: $20 / 20$ & $6 / 20$ & $\begin{array}{l}5 / 19 \\
\text { ( } 1 \text { flower not open, } 5 \\
\text { fertile corresponding } \\
\text { to } 6 \text { expected } \\
\text { fertile plants) }\end{array}$ & $\begin{array}{l}\text { Nuclear } \\
\text { contamination }\end{array}$ \\
\hline R1001B & $\begin{array}{l}\text { MsMs: } 1 / 8 \\
\text { Msms: } 4 / 8 \\
\text { msms: } 3 / 8\end{array}$ & $\begin{array}{l}\text { MsMs: } 2 / 20 \\
\text { Msms: } 9 / 20 \\
\text { msms: } 9 / 20\end{array}$ & $\begin{array}{l}\text { MsMs: } 1 / 8 \\
\text { Msms: } 4 / 8 \\
\text { msms: } 3 / 8\end{array}$ & $\begin{array}{l}\text { MsMs: } 1 / 8 \\
\text { Msms: } 4 / 8 \\
\text { msms: } 3 / 8\end{array}$ & $\begin{array}{l}\mathrm{N}: 17 / 20 \\
S: 3 / 20\end{array}$ & $20 / 20$ & $20 / 20$ & $\begin{array}{l}\text { Nuclear and } \\
\text { cytoplasmic } \\
\text { contamination }\end{array}$ \\
\hline R1201A & msms: $8 / 8$ & msms: $20 / 20$ & msms: $8 / 8$ & msms: $8 / 8$ & S: $20 / 20$ & $0 / 20$ & $0 / 20$ & Correct \\
\hline R1201B & $\begin{array}{l}\text { MsMs: } 2 / 8 \\
\text { Msms: } 4 / 8 \\
\text { msms: } 2 / 8\end{array}$ & $\begin{array}{l}\text { MsMs: } 6 / 20 \\
\text { Msms: } 11 / 20 \\
\text { msms: } 3 / 20\end{array}$ & $\begin{array}{l}\text { MsMs: } 2 / 8 \\
\text { Msms: } 4 / 8 \\
\text { msms: } 2 / 8\end{array}$ & $\begin{array}{l}\text { MsMs: } 2 / 8 \\
\text { Msms: } 4 / 8 \\
\text { msms: } 2 / 8\end{array}$ & $\begin{array}{l}\mathrm{N}: 9 / 20 \\
S: 11 / 20\end{array}$ & $18 / 20$ & $\begin{array}{l}16 / 17 \\
\text { ( } 1 \text { no pollen } \\
\text { corresponding to } \\
\text { expected sterile plant) }\end{array}$ & $\begin{array}{l}\text { Nuclear and } \\
\text { cytoplasmic } \\
\text { contamination }\end{array}$ \\
\hline R1401A & msms: $8 / 8$ & msms: $20 / 20$ & msms: $8 / 8$ & msms: $8 / 8$ & $\mathrm{~S}: 20 / 20$ & $0 / 20$ & $0 / 20$ & Correct \\
\hline
\end{tabular}

\subsection{Larger Scale Genotyping of Bulbs Planted for Seed Production in 2016}

Since molecular marker screening of reference onion lines in 2015 indicated some seedlings to have the wrong cytoplasmic male sterility genotypes and nuclear $M s$ allele contamination (Figure 1), all bulbs of four ARC onion lines (R101B, seed lot S03-2; R301A, seed lot S07-3; and R301B, seed lots S07-4 and S09-4), that were planted to produce seed in 2016, were genotyped in order to select individuals with the correct genotypes before flowering commenced in September 2016.

DNA were extracted from a total of 745 onion plants from the four breeding lines and the PCR molecular marker orfA501 and TaqMan ${ }^{\circledR}$ SNP genotyping assay A were applied. Figure 3 represents typical results of PCR molecular marker orfA501 products analysed on an Electro-Fast ${ }^{\circledR}$ stretch system. The Electro-Fast ${ }^{\circledR}$ Marker, loaded in additional wells of every second row, contains three bands of 1000, 500 and 200bp, respectively. The cytoplasmic and nuclear genotyping results of the four F1 hybrid parental lines are summarised in Table 2. 


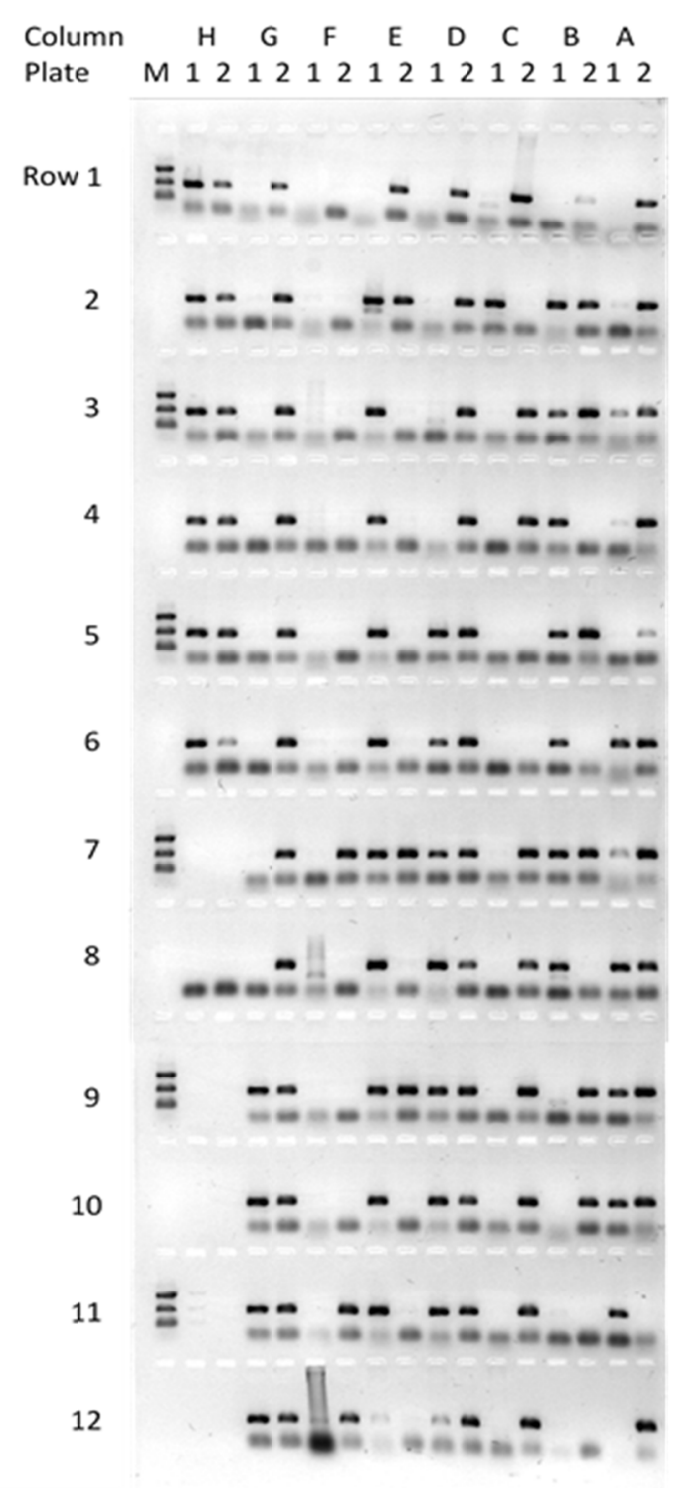

Figure 3. Example of Electro-Fast ${ }^{\circledR}$ stretch agarose gel electrophoresis of amplification products generated by PCR marker orfA501 on onion samples. Samples (180) of onion plants growing from bulbs in the field were genotyped. A fragment of 473bp is produced in cytoplasmic male sterile plants. Plate 1 or 2: 96-well PCR plate 1 or 2. Row 1 to 12: row of 96-well PCR plate. Column A to H: column of 96-well PCR plate. M: Electro-Fast ${ }^{\circledR}$ Marker was loaded in the first lane of every second row 
Table 2. Cytoplasmic and nuclear male sterile genotypes of four South African onion lines growing from bulbs in the field in 2016. PCR molecular marker orfA501 and TaqMan ${ }^{\circledR}$ SNP genotyping assay A were applied to 745 individuals to determine their cytoplasmic and nuclear male sterility genotypes. Results not as expected are indicated in italics

\begin{tabular}{|c|c|c|c|c|c|}
\hline \multirow{2}{*}{ Parental line } & \multirow{2}{*}{ Seed lot } & \multirow{2}{*}{$\begin{array}{l}\text { Nuclear genotype } \\
\text { TaqMan }{ }^{\circledR} \text { SNP } \\
\text { genotyping assay A }\end{array}$} & \multirow{2}{*}{$\begin{array}{l}\text { Cytoplasmic genotype } \\
\text { PCR marker orf } A 501\end{array}$} & \multicolumn{2}{|c|}{$\begin{array}{l}\text { Individuals with correct } \\
\text { combined genotype }\end{array}$} \\
\hline & & & & ratio & $\%$ \\
\hline R101B & S03-2 & $\begin{array}{l}\text { MsMs: } 0 / 8 \\
\text { Msms: } 4 / 8 \\
\text { msms: } 4 / 8\end{array}$ & $\begin{array}{l}\mathrm{N}: 4 / 60 \\
S: 56 / 60\end{array}$ & $1 / 60$ & $1.7 \%$ \\
\hline R301A & $\mathrm{S} 07-3^{*}$ & $\begin{array}{l}\text { MsMs: } 7 / 160 \\
\text { Msms: } 64 / 160 \\
\text { msms: } 88 / 160\end{array}$ & $\begin{array}{l}N: 18 / 160 \\
\mathrm{~S}: 142 / 160\end{array}$ & $80 / 160$ & $50.0 \%$ \\
\hline R301B & S07-4 & $\begin{array}{l}\text { MsMs: } 36 / 354 \\
\text { Msms: } 83 / 354 \\
\text { msms: } 235 / 354\end{array}$ & $\begin{array}{l}\mathrm{N}: 308 / 354 \\
S: 46 / 354\end{array}$ & $207 / 354$ & $58.5 \%$ \\
\hline R301B & S09-4 & $\begin{array}{l}\text { MsMs: } 8 / 171 \\
\text { Msms: } 77 / 171 \\
\text { msms: } 86 / 171\end{array}$ & $\begin{array}{l}\text { N: } 94 / 171 \\
S: 77 / 171\end{array}$ & $48 / 171$ & $28.1 \%$ \\
\hline
\end{tabular}

Note. ${ }^{*}$ One individual plant grown from seed lot $\mathrm{S} 07-3$ did not yield any results after three attempts with TaqMan® SNP genotyping assay A.

\section{Discussion}

\subsection{Preliminary Testing of PCR Molecular Markers for Determination of Male Sterility Genotypes}

The results indicated that markers 5'cob, orfA501, IGS and jnurf13 were useful for South African parental onion line genotyping, while orf 725 may give inconclusive results and cob-type 2 failed completely to produce the expected fragment. According to Sato (1998), an insertion into the upstream region of the mitochondrial cob gene in cytoplasmic male sterile (S) mitotypes can be amplified with specific flanking oligonucleotides to produce a $414 \mathrm{bp}$ fragment. A $180 \mathrm{bp}$ fragment is amplified from the $\mathrm{N}$-cytoplasm with a $\mathrm{N}$-specific primer and the same common reverse primer. The use of the three primers $(S-, N$-specific and common reverse primer) together in the same PCR is recommended by Engelke et al. (2003) so that sub-stoichiometric amounts of the 414bp $S$-fragment is not amplified in $N$-cytoplasm. Both fragments are amplified in $S$-cytoplasm. In this study, faint 180bp amplification products of the normal cob gene were obtained in all samples, although it was stronger in samples designated as $N$ (Figure 1, $5^{\prime} \mathrm{cob}$ ). The $414 \mathrm{bp}$ fragment was only observed in samples designated as $S$, as expected, and was therefore a useful marker.

The orfA501 marker is based on an insertion in the mitochondrial sequence of chives that forms an open reading frame (orfA501) (Engelke et al., 2003). Primers which span nearly the complete orfA501 amplify a 473bp fragment in both $T$ - and $S$-cytoplasm in onion, but nothing in $N$-cytoplasm. It is reported to produce weak amplification from $\mathrm{N}$-cytoplasm, so may give inconclusive results, but in this study orfA501 proved useful as a presence/absence marker for cytoplasmic male sterility.

The third useful cytoplasmic marker, IGS, is based on a polymorphic sequence in the intergenic spacer (IGS) between $\operatorname{trnT}$ and $\operatorname{trnL}$ of the chloroplast genome of $N$ - and $S$-cytoplasm types (Cp-cytotypes; Havey, 1995). Kim (2014) designed a new primer pair on the flanking sequences of the 89bp indel reported by Havey (1995) in order to achieve an improved resolution. A 388bp IGS fragment is amplified from the $N$-cytoplasm, and the smaller 299bp fragment from $S$-cytoplasm. In this study, the $I G S$ marker gave very conclusive results. Due to the small size difference between the $N$ and $S I G S$ PCR fragments (388 and 299bp, respectively), long electrophoresis times were required to separate them on $1.5 \%$ agarose gels.

There is a chloroplastic $y c f 2$ gene fragment integration upstream of the mitochondrial cob gene (Kim \& Yoon, 2010). This cob-type 2 is predominant only in the CMS-S cytotype. Primers specific to the $y c f 2$ and $c o b$ sequences were combined to amplify an $852 \mathrm{bp}$ fragment only from $S$-cytoplasm. This marker was unfortunately not effective in detecting the $852 \mathrm{bp} S$-specific fragment from ARC onion parental lines and was excluded from the rest of this study. 
jnurf13 is a codominant marker in linkage disequilibrium with the restorer-of-fertility locus of both $S$ and $T$ cytoplasmic male sterility systems. It is based on a $12 \mathrm{bp}$ indel identified by Kim (2014) in sequences flanking the jnurf12 marker, a fragment initially tagged by Yang et al. (2013). The smaller fragment (229bp) is linked with the $m s$-allele, while the $241 \mathrm{bp}$ product is produced from $M s$-genotypes. In this study, the jnurfl3 marker was very successful in distinguishing between the dominant and recessive alleles of the nuclear restorer-of-fertility gene $(M S)$ in four selected onion lines. Since it is a codominant marker, the heterozygous genotypes (Msms) could be distinguished from the homozygous dominant (MsMs) genotypes. After jnurf13, Huo, Liu, Yang, and $\mathrm{Wu}$ (2015) developed the multiplex PCR-based co-dominant marker AcSKP1 that is in complete linkage disequilibrium with the $M s / m s$ locus. Its coding sequence is predicted to encode a putative S-phase kinase-associated protein 1, which may have functions in pollen development and male fertility in onion according to studies with Arabidopsis homologs. The jnurf13 and AcSKP1 markers are considered as the same locus from a breeding perspective, since both originated from the study performed by Yang et al. (2013).

\subsection{TaqMan ${ }^{\circledR}$ SNP Genotyping Assays for Determination of Male Sterility Genotypes}

The advantages of TaqMan ${ }^{\circledR}$ SNP genotyping assays are their ease of use, reproducibility, small template requirement and rapid high throughput genotyping of nuclear male sterility genotypes in large onion breeding populations. In this study, three SNP markers linked to the Ms locus (Duangjit et al., 2013; Havey, 2013) were successfully converted into TaqMan ${ }^{\circledR}$ SNP genotyping assays and used for the first time to discriminate between the dominant and recessive alleles of the nuclear restorer-of-fertility gene (Ms). TaqMan ${ }^{\circledR}$ SNP genotyping assay B (isotig30856_1351; Figure 2, pink) gave the best separated groups corresponding to homozygous recessive, heterozygous and homozygous dominant genotypes ( $m s m s$, Msms and MsMs) in the scatterplot analysis performed through the Rotorgene software. The advantage of the successful TaqMan ${ }^{\circledR}$ SNP genotyping assays, instead of the PCR molecular marker jnurf 13, is the speed of obtaining results. Analysis of 68 samples can be performed in 2 hours, while PAGE electrophoresis of jnurf 13 PCR products on a large gel system at the ARC is currently limited to 34 lanes per two days. After these nuclear markers were selected and applied during this study in conjunction with jnurf13, the multiplex PCR-based co-dominant AcSKP1 marker was developed (Huo et al., 2015). Since AcSKP1 amplifies larger PCR products than jnurf13, AcSKP1 can be detected with simple agarose gel electrophoresis instead of polyacrylamide gels. This marker was successfully applied subsequent to this study to assign nuclear genotypes (results not shown), and proves to be cheaper than TaqMan SNP ${ }^{\circledR}$ assays, but results are not generated as rapidly.

\subsection{Correlation of Cytoplasmic and Nuclear Genotype With the Pollen Fertility Phenotype of Onion Breeding Pairs}

R601A and R601B are all nuclear msms (Table 1), if TaqMan ${ }^{\circledR}$ SNP genotyping assay B results are disregarded (see Discussion 5.4). The A-line is cytoplasmic $S$ in all but 1 of the 20 samples (R601A_13), and the B-line has the correct $N$ genotype, even though one sample (R601B_6) tested $S$ with only one of the four cytoplasmic markers (orfA501). No pollen was observed on sample R601A_13. It was expected to be fertile since it was cytoplasmic $N$, genotyped with four markers. The genotypes of this male sterile-maintainer pair are possibly correct since only a rare event of cytoplasm contamination was detected. It could have been caused by mixing with a wrong bulb during harvesting.

All samples of R801A were cytoplasmic $S$ and nuclear $m s m s$ with all markers tested. All the plants were expected to be sterile and indeed no pollen was detected on all replicates. All samples of R801B were cytoplasmic $N$ and nuclear $m s m s$ with all markers tested. All the plants were expected to be fertile and indeed pollen was detected on all replicates. R801A can be used as is for F1 hybrid seed production, while A-line seed can be produced successfully with R801B.

Both R1001A and R1001B were contaminated with nuclear Ms. All samples of the A-line were cytoplasmic $S$, but the B-line was contaminated with three $S$ samples. The nuclear genotype predicted the fertility of the pollen correctly for all but one R1001A plant, due to the nuclear Msms genotype, since their cytoplasm were all sterile ( $5 / 6$ fertile). This plant showing the discrepancy could have been phenotyped incorrectly due to flower maturity at the time of screening. Otherwise, it should be considered that this male-fertile plant was misclassified as male-sterile due to the dominant $M s$ allele showing reduced penetrance (Melgar \& Havey, 2010). They suggested that the phenotype of the onion plant may not be the same as the genotype when the plant has the MsMs or Msms genotype, requiring that male-fertility restoration be scored over years to confidently assign genotypes at the $M s$ locus.

R1001B is contaminated with both the nuclear $M s_{-}$allele and $S$ cytoplasm. The three plants having sterile cytoplasm were contaminated with the $M s_{-}$allele in all three cases, rendering them fertile and therefore pollen 
was detected on all 20 replicates. The correct genotype of R1001B cannot be recovered and cannot be used as is to produce A-line seed, even if the A-line was correct.

All samples of R1201A were cytoplasmic $S$ and nuclear $m s m s$ with all markers tested. All the plants were expected to be sterile and indeed no pollen was detected on all 20 replicates. The A line is therefore correct, but R1201B was contaminated both with the nuclear $M s_{-}$allele and $S$ cytoplasm, rendering it unsuitable for a pure maintainer line. Even though there was quite a high number of cytoplasmic $S$ plants, their sterility was being masked by contamination by the nuclear $M s$ allele. The nuclear genotype ( $m s m s$ ) predicted that only two plants should have sterile pollen (R1201B_2 and 11). Unfortunately, the pollen status of only one of them was phenotyped successfully, being plant \#11 having no pollen. The other flower from plant \#2 was not open yet when fertility was scored. For recovery of the B-line, R1201B plants, that are not only cytoplasmic $N$, but also nuclear $m s m s$, which are $15-25 \%$ of the population (3/20 and 2/8 plants tested were $m s m s$, respectively), should be selected for propagating pure maintainer seed.

All samples of R1401A were cytoplasmic $S$ and nuclear $m s m s$ with all markers tested. All the plants were expected to be sterile and indeed no pollen was detected on all 20 replicates. Unfortunately, the B-line of this breeding pair was lost when all the bulbs rotted during storage and, consequently, the genotyping and phenotyping could not be done.

\subsection{Discrepancy Between Markers for Nuclear Genotype: TaqMan ${ }^{\circledR}$ SNP Assay B vs Assay A, C and jnurf13}

A discrepancy was detected in the nuclear genotypes determined with TaqMan ${ }^{\circledR}$ SNP genotyping assays A, B, C and marker jnurfl 3 of onion breeding pair R601A and B. In these two lines, SNP assay A, C and jnurf13 showed all samples to have the msms genotype, but SNP assay B detected heavy $M s$ allele contamination (Table 1). All the other onion breeding lines tested (S03-2, R801, R1201, R1001, R1401) were genotyped identically with the three SNP assays and jnurf 13. The discrepancy is therefore an isolated phenomenon to the R601 breeding pair, and not observed among other onion parental lines.

Phenotypic observations of plant male fertility (pollen-containing or sterile) cannot be used for R601B plants to resolve the correct marker to use that is more closely linked to the restorer-of-male fertility gene, since it is a maintainer line with normal cytoplasm, and all plants are therefore male fertile. But, since the male sterile R601A plants were all without pollen (observed fertility column in Table 1), it is proposed that TaqMan ${ }^{\circledR}$ SNP assays $\mathrm{A}$ and $\mathrm{C}$ and jnurf 13 are giving the correct genotype (msms).

The recessive $m s$ allele showed 11,13 and $15.7 \%$ recombination with the SNPs detected by assays A, B and C, respectively, among random plants from three open-pollinated populations of onion (Duangjit et al., 2013; Havey, 2013). The SNP detected by assay B (isotig30856_1351, Appendix B) is therefore further away from the Ms/ms locus than that detected by assay A (isotig34671_610), with a higher possibility of crossover between them. It was therefore suggested that recombination occurred between SNP isotig30856_1351 and the Ms/ms locus in this onion breeding pair, rendering the TaqMan ${ }^{\circledR}$ SNP genotyping assay B an unsuitable marker for $m s$ genotyping the parental lines of R601. If the SNP isotig29186_1830 (detected by assay C), which is even further away from the $M s / m s$ locus than isotig30856_1351 (detected by assay B), is located at the same side of the crossover than SNP isotig30856_1351, it should yield the same genotype as assay B. To test this hypothesis, eight replicates of each onion line were genotyped with TaqMan ${ }^{\circledR}$ SNP genotyping assay C (isotig29186_1830). It was found that assay $\mathrm{C}$ genotypes corresponded to that of TaqMan ${ }^{\circledR}$ SNP assay A and jnurf 13 genotypes in all cases, including line R601 (Table 1). It can be concluded, therefore, that SNP isotig29186_1830 (detected by assay C) is located at the opposite side of the $M s / m s$ locus, and is unaffected by the crossover between the $M s / m s$ locus and SNP isotig30856_1351 (detected by assay B).

\subsection{Larger Scale Genotyping of Bulbs Planted for Seed Production in 2016}

When bulbs of four ARC onion lines (R101B, seed lot S03-2; R301A, seed lot S07-3; and R301B, seed lots S07-4 and S09-4) were genotyped (Table 2), the TaqMan® SNP genotyping assay A was chosen because of the possible discrepancy of the SNP genotyping assay B results with the true $M s / m s$ genotype.

PCR molecular marker orfA501 proved useful in this study as a presence/absence high-throughput marker for cytoplasmic male sterility. The production of a PCR fragment of $473 \mathrm{bp}$ is associated with $S$ cytoplasm, and the absence with $N$ cytoplasm. PCR products of this marker was run on a high density agarose gel of the Electro-Fast ${ }^{\circledR}$ stretch system (Figure 3), which is intended for high throughput with samples that contain a small number of signals. This system also has the advantages of minimising buffer use and reducing processing time. Because it has a shorter separation distance than low throughput gels, separation of differently sized fragments 
with long electrophoresis times are not possible. Results of marker orfA501 could be obtained much faster with 2 $\times 96$ samples loaded with a multi-channel pipette and separated within $30 \mathrm{~min}$.

For line S07-3, S07-4 and S09-4 the percentage of plants with the correct combination of cytoplasmic and nuclear male sterility genotypes ranged from 28.1 to $58.5 \%$ (Table 2). The incorrect plants were removed and the seed production will proceed with correct plants resulting in pure maintainer and male sterile seed for future use. For line S03-2 a mere 1.7\% (1 out of 60) plants were correct and thus the seed production was aborted.

\section{Conclusion}

A combination of PCR molecular markers and three TaqMan ${ }^{\circledR}$ SNP genotyping assays were used successfully to genotype cytoplasmic and nuclear male sterility of five South African onion breeding pairs, as well as applied on a large scale to two more breeding pairs. PCR marker orfA501 proved useful as a high-throughput presence/absence marker for cytoplasmic male sterility, which can be run on a high density agarose gel at high throughput without having to separate differently sized PCR fragments with long electrophoresis times. TaqMan ${ }^{\circledR}$ SNP genotyping assays were superior to the jnurf13 nuclear marker in terms of rapid throughput.

The mismatching TaqMan ${ }^{\circledR}$ SNP genotyping assays A, B, C and jnurf 13 marker results are shared between the male sterile-maintainer pair of the R601 onion breeding line. This discrepancy is an isolated phenomenon to this breeding pair, and not observed among any other South African onion breeding lines tested. It is suggested that recombination occurred between SNP isotig30856_1351 and the Ms/ms locus in this onion breeding pair.

High rates of contamination with the dominant restorer-of-fertility $M s$ allele of both A and B onion parent lines were detected. Cytoplasm types $(S$ or $N$ ) were as expected only for a few lines. Only the R801 breeding pair seems to have its nuclear and cytoplasmic male sterility genotypes in order. Genotype data could successfully be used to rogue plants with the wrong genotypes, enabling the recovery of the correct genotypes of male sterile-maintainer parental lines used in hybrid seed production, and facilitating the production of the correct seed for commercialization.

PCR and TaqMan ${ }^{\circledR}$ genotyping can be done in two days, including genomic DNA isolation from any leaf or bulb material. The breeder does not have to wait for seed production, bulb generation and subsequent evaluation of the male sterility status at flowering time. Using genetic screening techniques enabled the onion breeding program to reduce the time to identify the correct genotype of male sterile and maintainer-lines from two years to one month, gave accurate genotypic information that was not previously available and proved to be useful on a larger scale.

\section{References}

Brewster, J. L. (2008). Onions and other vegetable alliums. CABI, Cambridge. https://doi.org/10.1079/9781 845933999.0000

Cho, K.-S., Yang, T.-J., Hong, S.-Y., Kwon, Y.-S., Woo, J.-G., \& Park, H.-G. (2006). Determination of cytoplasmic male sterile factors in onion plants (Allium cepa L.) using PCR-RFLP and SNP markers. Molecules and Cells, 21(3), 411-417.

Duangjit, J., Bohanec, B., Chan, A. P., Town, C. D., \& Havey, M. J. (2013). Transcriptome sequencing to produce SNP-based genetic maps of onion. Theoretical and Applied Genetics, 126, 2093-2101. https://doi.org/10.1007/s00122-013-2121-x

Engelke, T., Terefe, D., \& Tatlioglu, T. (2003). A PCR-based marker system monitoring CMS-(S), CMS-(T) and (N)-cytoplasm in the onion (Allium cepa L.). Theoretical and Applied Genetics, 107(1), 162-167. https://doi.org/10.1007/s00122-003-1230-3

Gai, S. P., \& Meng, X. D. (2010). Application of molecular markers linking to cytoplasmic male sterile loci to assist maintainer line selection and their selection efficiency in Welsh onion (Allium fistulosum L.). Agricultural Sciences in China, 9(11), 1571-1576. https://doi.org/10.1016/S1671-2927(09)60253-4

Havey, M. J. (1995). Cytoplasmic determinations using the polymerase chain reaction to aid in the extraction of maintainer lines from open-pollinated populations of onion. Theoretical and Applied Genetics, 90, 263-268. https://doi.org/10.1007/BF00222212

Havey, M. J. (2000). Diversity among male-sterility-inducing and male-fertile cytoplasms of onion. Theoretical and Applied Genetics, 101, 778-782. https://doi.org/10.1007/s001220051543 
Havey, M. J. (2013). Single nucleotide polymorphisms in linkage disequilibrium with the male-fertility restoration (Ms) locus in open-pollinated and inbred populations of onion. Journal of the American Society for Horticultural Science, 138(4), 306-309.

Huo, Y., Liu, B. J., Yang, Y. Y., \& Wu, X. (2015). AcSKP1, a multiplex PCR-based co-dominant marker in complete linkage disequilibrium with the male-fertility restoration $(M s)$ locus, and its application in open-pollinated populations of onion. Euphytica, 204(3), 711-722. https://doi.org/10.1007/s10681-015$1374-7$

Kim, S. (2014). A codominant molecular marker in linkage disequilibrium with a restorer-of-fertility gene (Ms) and its application in reevaluation of inheritance of fertility restoration in onions. Molecular Breeding, 34, 769-778. https://doi.org/10.1007/s11032-014-0073-8

Kim, S., \& Yoon, M.-K. (2010). Comparison of mitochondrial and chloroplast genome segments from three onion (Allium cepa L.) cytoplasm types and identification of a trans-splicing intron of cox2. Current Genetics, 56, 177-188. https://doi.org/10.1007/s00294-010-0290-6

Kim, S., Lee, E.-T., Cho, D. Y., Han, T., Bang, H., Patil, B. S., .. Yoon, M.-K. (2009). Identification of a novel chimeric gene, orf725, and its use in development of a molecular marker for distinguishing among three cytoplasm types in onion (Allium cepa L.). Theoretical and Applied Genetics, 118, 433-441. https://doi.org/ 10.1007/s00122-008-0909-x

Melgar, S., \& Havey, M. J. (2010). The dominant Ms allele in onion shows reduced penetrance. Journal of the American Society for Horticultural Science, 135, 49-52.

Moyo, M., Amoo, S. O., Bairu, M. W., Finnie, J. F., \& Van Staden, J. (2008). Optimising DNA isolation for medicinal plants. South African Journal of Botany, 74, 771-775. https://doi.org/10.1016/j.sajb.2008.07.001

Murray, M. G., \& Thompson, W. F. (1980). Rapid isolation of high molecular weight plant DNA. Nucleic Acids Research, 8(19), 4321-4325. https://doi.org/10.1093/nar/8.19.4321

Sato, Y. (1998). PCR amplification of CMS-specific mitochondrial nucleotide sequences to identify cytoplasmic genotypes of onion (Allium cepa L.). Theoretical and Applied Genetics, 96(3-4), 367-370. https://doi.org/ $10.1007 / \mathrm{s} 001220050750$

Sharma, P., \& Purohit, S. D. (2012). An improved method of DNA isolation from polysaccharide rich leaves of Boswellia serrata Roxb. Indian Journal of Biotechnology, 11, 67-71.

Yang, Y. Y., Huo, Y. M., Miao, J., Liu, B. J., Kong, S. P., Gao, L. M., ... Wu, X. (2013). Identification of two SCAR markers co-segregated with the dominant $M s$ and recessive $m s$ alleles in onion (Allium cepa L.). Euphytica, 190, 267-277. https://doi.org/10.1007/s10681-012-0842-6 


\section{Appendix A}

PCR Molecular Markers Useful for Discrimination Between Onion Cytoplasmic and Nuclear Male Sterility Genotypes

PCR primer sequences and their expected product sizes obtained from different cytoplasmic and nuclear genotypes are indicated.

\begin{tabular}{|c|c|c|c|c|c|c|c|c|c|}
\hline \multirow[t]{2}{*}{ Marker } & \multirow[t]{2}{*}{$\begin{array}{l}\text { Gene } \\
\text { region }\end{array}$} & \multirow[t]{2}{*}{ Organelle } & \multirow[t]{2}{*}{ Reference } & \multirow[t]{2}{*}{$\begin{array}{l}\text { Primer } \\
\text { name }\end{array}$} & \multirow[t]{2}{*}{$\begin{array}{l}\text { PCR primer nucleotide } \\
\text { sequence (5'-3') }\end{array}$} & \multirow[t]{2}{*}{$\begin{array}{l}\mathrm{Tm} \\
{ }^{\circ} \mathrm{C}\end{array}$} & \multicolumn{3}{|c|}{$\begin{array}{l}\text { Expected amplification } \\
\text { product at each } \\
\text { genotype (bp) }\end{array}$} \\
\hline & & & & & & & $\mathbf{N}$ & $\mathbf{T}$ & $\mathbf{S}$ \\
\hline \multirow[t]{13}{*}{ Cytoplasmic } & 5 'cob & Mitochondrion & Sato, 1998 & Scob-F & GTCCAGTTCCTATAGAACCTATCACT & 57 & 180 & 180 & $180+414$ \\
\hline & & & & Ncob-F & TCTAGATGTCGCATCAGTGGAATCC & & & & \\
\hline & & & & cob-R & СTTTTCTATGGTGACAACTCCTCTT & & & & \\
\hline & OrfA501 & Mitochondrion & Engelke & orfA501-F & ATGGCTCGCCTTGAAAGAGAGC & 60 & weak & 473 & 473 \\
\hline & & & et al., 2003 & orfA501-R & CCAAGCATTTGGCGCTGAC & & & & \\
\hline & Orf 725 & Mitochondrion & Kim et al., & $\operatorname{cox} 1-\mathrm{F}$ & CATAGGCGGGCTCACAGGAATA & 62 & 833 & $833+628$ & 628 \\
\hline & & & 2009 & orf725-R & AATCCTAGTGTCCGGGGTTTCT & & & & \\
\hline & & & & $\operatorname{cox} 1-\mathrm{R}$ & CAGCGAACTTTCATTCTTTCGC & & & & \\
\hline & $I G S$ & Chloroplast & Kim, 2014 & Cpcyto-F & GGGTCGATATTCTTCCTTATTGTTT & 55 & 388 & 388 & 299 \\
\hline & (modified) & & & Cpcyto-R & 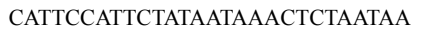 & & & & \\
\hline & Cob-type 2 & Mitochondrion & Kim \& & cob-F2 & CGGAGCGAAAAGGGTTTTCCATGAGAT & 58 & & & 852 \\
\hline & & & Yoon, 2010 & cob-R2 & TTGTATGTATGCCCGATCCA & & & & \\
\hline & & & & & & & Ms & $m s$ & \\
\hline \multirow[t]{2}{*}{ Nuclear } & jnurf13 & Nucleus & Kim, 2014 & jnurf13-F & TGCAAGCTTGGAACTTACGC & 55 & 241 & 229 & \\
\hline & & & & jnurf13-R & TTGCCAAAGGTTGCAATACA & & & & \\
\hline
\end{tabular}

\section{Appendix B}

TaqMan® ${ }^{\circledR}$ SNP Genotyping Assays Were Designed for Three Sequences Containing SNPs in Linkage Disequilibrium With the ms Allele of Onion (Duangjit et al., 2013; Havey, 2013)

Primer binding sites are italicized and highlighted with yellow or green for the forward and reverse primer, respectively. Probe binding areas are underlined. The second nucleotide in brackets [ ] is coupled with the recessive $m s$ allele. The VIC reporter dye was coupled to the probe binding to the dominant $M s$ allele (first nucleotide in brackets [ ]), and the FAM reporter dye was coupled to the probe binding to the recessive $m s$ allele (second nucleotide in brackets []).

SNP assay A
Auxin-induced protein
isotig34671_610
TGCAGCAACTGTCAAANATTGGATCAATCCGAAAAAGCAAGTGAAGGCTGTTACAGAAAG[C/T]CTGGCTGGAT
ACTGCTTTAGCACTGGAACCTGAAGAACCATCCATCCAGACCAGGCGAGA
SNP assay B
Pentatricopeptide repeat-containing protein
isotig30856_1351
GTTGAAGCACTATGGATGCATGGCTGATCTGTATGGACGGTCTGGATTGGTTGATGAGGC[G/A]aTGGAGATGAT
AGAAGGGATGCCGATGAAGGCGGATGAGTATGTGTGGGGAGCGGTGCTT
SNP assay C
RNA polymerase II C-terminal domain phosphatase like 2
isotig29186_1830
GAATGATCCGAGTAAGAACAATGTTACGATCTGACAATCGAATAACCGGACGAGAAGCAC[A/G]GgTTTGTGCA
CCAGGTAATTGCAAGACTTGTTCGTTTTGTGCAGATACTGGCATCCCATT




\section{Appendix C}

\section{Summary of Materials Used}

C.1 Genomic DNA isolation

Carborundum (180 grit)

PVPP

CTAB/PVP isolation buffer: 2.5\% (w/v) CTAB; $2 \mathrm{M} \mathrm{NaCl} ; 20 \mathrm{mM}$ EDTA; $100 \mathrm{mM}$ Tris (pH 8.0); 1\% PVP (Sigma PVP-40); 0.2\% (v/v) $\beta$-mercaptoethanol;

chloroform: isoamyl alcohol (CIAA, 24:1)

$10 \% \mathrm{CTAB} / 0.7 \mathrm{M} \mathrm{NaCl}$ solution

ethanol at room temperature

high salt TE (TE with $1 \mathrm{M} \mathrm{NaCl}$ )

RNaseA (10 mg/ml; $2.5 \mu l$ per extract)

isopropanol at room temperature

$70 \%$ ethanol

TE buffer

C.2 PCR molecular markers for determination of cytoplasmic and nuclear male sterile genotypes

PCR primers (oligonucleotides) indicated in Appendix A

TaKaRa Taq (TAKARA BIO INC., Otsu, Shiga, Japan) with buffer, $\mathrm{MgCl}_{2}$ and dNTP mix

200 ul PCR tubes and 96-well PCR plates

Thermocycler (PCR machine)

agarose $/ 0.5 \times$ TAE gel (containing $0.1 \mu \mathrm{g} / \mathrm{ml}$ Ethidium Bromide)

Ethidium Bromide $(10 \mathrm{mg} / \mathrm{ml})$

UV light and gel documentation system

MWM VI (Roche Diagnostics)

Electro-Fast ${ }^{\circledR}$ stretch agarose gel $(1.5 \% \mathrm{w} / \mathrm{v})$ (ABgene)

ReddyRun ${ }^{\mathrm{TM}}$ loading dye (ABgene)

Electro-Fast ${ }^{\circledR}$ marker (ABgene; $25 \mathrm{ng} / \mu 1,3 \mu \mathrm{l}$ per well)

denaturing $6 \% \mathrm{PAGE} / 8 \mathrm{M} \mathrm{Urea} / 1 \times \mathrm{TBE}$ gel electrophoresis

silver nitrate staining of PAGE gel

10bp DNA ladder (Roche)

MWM V (Roche Diagnostics)

C.3 TaqMan ${ }^{\circledR}$ SNP genotyping assay for the detection of SNP linked to the nuclear male sterility factor

TaqMan ${ }^{\circledR}$ SNP genotyping assay mix (Applied Biosystems, cat. nu 4332077) as in Appendix B

TaqMan ${ }^{\circledR}$ genotyping master mix (Applied Biosystems, cat. nu 4371355)

Real-time PCR machine (in this case: Rotorgene-3000 (Corbett Life sciences, Qiagen))

$100 \mu$ l Rotorgene tubes

\section{Copyrights}

Copyright for this article is retained by the author(s), with first publication rights granted to the journal.

This is an open-access article distributed under the terms and conditions of the Creative Commons Attribution license (http://creativecommons.org/licenses/by/4.0/). 